PROCEEDINGS OF THE

AMERICAN MATHEMATICAL SOCIETY

Volume 127, Number 12, Pages 3591-3592

S 0002-9939(99)05462-3

Article electronically published on July 8, 1999

\title{
CONVOLUTION WITH AFFINE ARCLENGTH MEASURES IN THE PLANE
}

\author{
DANIEL M. OBERLIN
}

(Communicated by Christopher D. Sogge)

\begin{abstract}
We obtain an estimate for the $L^{3 / 2,1}\left(\mathbb{R}^{2}\right)-L^{3}\left(\mathbb{R}^{2}\right)$ norm of a certain convolution operator.
\end{abstract}

Let $\phi$ be real-valued and smooth on an interval $(a, b) \subseteq \mathbb{R}$. Define the measure $\lambda$ on $\mathbb{R}^{2}$ by

$$
\int_{\mathbb{R}^{2}} f d \lambda=\int_{a}^{b} f(t, \phi(t))\left|\phi^{\prime \prime}(t)\right|^{1 / 3} d t .
$$

We are interested in the $L^{p}\left(\mathbb{R}^{2}\right)-L^{q}\left(\mathbb{R}^{2}\right)$ mapping properties of the operator given by convolution with $\lambda$. The study of this operator was initiated by Drury ([D]), who used complex interpolation and certain integral estimates to obtain the optimal result

$$
\|\lambda * f\|_{3} \leq C\|f\|_{3 / 2}
$$

when $\phi$ satisfies certain regularity conditions. Drury's approach was refined and extended by Choi in $[\mathrm{C}]$. The purpose of this note is to prove an almost-sharp estimate for a large class of functions $\phi$ :

Theorem. Suppose $\phi^{\prime \prime}>0$ and $\phi^{(3)} \geq 0$ on $(a, b)$. Then

$$
\left\|\lambda * \chi_{E}\right\|_{3} \leq 12^{1 / 3}\left\|\chi_{E}\right\|_{3 / 2}
$$

for any measurable $E \subseteq \mathbb{R}^{2}$.

Proof. Define $\gamma(s)=(s, \phi(s))$. Inequality (1) is equivalent to

$$
\int_{\mathbb{R}^{2}} \int_{a}^{b} \int_{a}^{b} \int_{a}^{b} \prod_{1}^{3}\left[\chi_{E}\left(x-\gamma\left(s_{i}\right)\right) \phi^{\prime \prime}\left(s_{i}\right)^{1 / 3}\right] d s_{1} d s_{2} d s_{3} d x \leq 12|E|^{2}
$$

and so will follow from

$$
\int_{\mathbb{R}^{2}} \int_{a}^{b} \int_{s_{3}}^{b} \int_{s_{3}}^{b} \prod_{1}^{3}\left[\chi_{E}\left(x-\gamma\left(s_{i}\right)\right) \phi^{\prime \prime}\left(s_{i}\right)^{1 / 3}\right] d s_{1} d s_{2} d s_{3} d x \leq 4|E|^{2} .
$$

Received by the editors February 16, 1998.

1991 Mathematics Subject Classification. Primary 42B15.

The author was partially supported by a grant from the National Science Foundation.

(C)1999 American Mathematical Society 
By Fubini's theorem this is equivalent to

$$
\int_{\mathbb{R}^{2}} \chi_{E}(x) \int_{a}^{b}\left(\int_{t}^{b} \chi_{E}(x+\gamma(t)-\gamma(s)) \phi^{\prime \prime}(s)^{1 / 3} d s\right)^{2} \phi^{\prime \prime}(t)^{1 / 3} d t d x \leq 4|E|^{2} .
$$

Thus, it is enough to establish the inequality

$$
\int_{a}^{b}\left(\int_{t}^{b} \chi_{E}(\gamma(t)-\gamma(s)) \phi^{\prime \prime}(s)^{1 / 3} d s\right)^{2} \phi^{\prime \prime}(t)^{1 / 3} d t \leq 4|E| .
$$

The convexity of the graph of $\phi$ shows that the change of variables

$$
(s, t) \rightarrow \gamma(s)-\gamma(t)=(s-t, \phi(s)-\phi(t))
$$

is one-to-one. Thus

$$
\int_{a}^{b} \int_{a}^{b} \chi_{E}(\gamma(t)-\gamma(s))\left|\phi^{\prime}(s)-\phi^{\prime}(t)\right| d s d t \leq|E|
$$

and (2) will follow from the inequality

$$
\phi^{\prime \prime}(t)^{1 / 3}\left(\int_{t}^{b} \chi_{A}(s) \phi^{\prime \prime}(s)^{1 / 3} d s\right)^{2} \leq 4 \int_{t}^{b} \chi_{A}(s)\left(\phi^{\prime}(s)-\phi^{\prime}(t)\right) d s
$$

if $A \subseteq(t, b)$. To prove (3) we let $\left|A_{u}\right|$ stand for the (one-dimensional) Lebesgue measure of $A \cap(u, b)$ whenever $t \leq u \leq b$. Then

$$
\int_{t}^{b} \chi_{A}(s)\left(\phi^{\prime}(s)-\phi^{\prime}(t)\right) d s=\int_{t}^{b} \chi_{A}(s) \int_{t}^{s} \phi^{\prime \prime}(u) d u d s=\int_{t}^{b} \phi^{\prime \prime}(u)\left|A_{u}\right| d u .
$$

Also

$$
\begin{aligned}
& \int_{t}^{b} \chi_{A}(s) \phi^{\prime \prime}(s)^{1 / 3} d s=\int_{t}^{b} \chi_{A}(s) \phi^{\prime \prime}(s)^{1 / 3}\left|A_{s}\right|^{1 / 3}\left|A_{s}\right|^{-1 / 3} d s \\
& \leq\left(\int_{t}^{b} \chi_{A}(s) \phi^{\prime \prime}(s)\left|A_{s}\right| d s\right)^{1 / 3}\left(\int_{t}^{b} \chi_{A}(s)\left|A_{s}\right|^{-1 / 2} d s\right)^{2 / 3} .
\end{aligned}
$$

Thus, it follows from (4) that

$$
\left(\int_{t}^{b} \chi_{A}(s) \phi^{\prime \prime}(s)^{1 / 3} d s\right)^{3} \leq\left(\int_{t}^{b} \chi_{A}(s)\left(\phi^{\prime}(s)-\phi^{\prime}(t)\right) d s\right)\left(\int_{t}^{b} \chi_{A}(s)\left|A_{s}\right|^{-1 / 2} d s\right)^{2} .
$$

If $0 \leq \rho \leq|A|$, then $\left|\left\{s \in A:\left|A_{s}\right| \leq \rho\right\}\right|=\rho$, and so

$$
\int_{t}^{b} \chi_{A}(s)\left|A_{s}\right|^{-1 / 2} d s=\int_{0}^{|A|} y^{-1 / 2} d y=2|A|^{1 / 2} .
$$

With this and the fact that $\phi^{\prime \prime}$ is nondecreasing, (5) yields (3) to complete the proof.

\section{REFERENCES}

[C] Y. Choi, Convolution operators with affine arclength measures on plane curves, J. Korean Math. Soc. 36 (1999), 193-207. CMP 99:09

[D] S. W. Drury, Degenerate curves and harmonic analysis, Math. Proc. Camb. Phil. Soc. 108 (1990), 89-96. MR 91h:42021

Department of Mathematics, Florida State University, Tallahassee, Florida 32306 4510

E-mail address: oberlin@math.fsu.edu 